\title{
GKV-Finanzierungsreform: schwarz-gelbe Irrwege statt gezielter Problemlösungen
}

„Für ein gerechtes, soziales, stabiles, wettbewerbliches und transparentes Gesundheitssystem" - fürwahr eine anspruchsvolle Zielsetzung, die sich die schwarz-gelbe Bundesregierung für ihre Reform der Finanzierung der gesetzlichen Krankenversicherung gestellt hat. Dabei ist die Liste der für die Reform reklamierten Attribute noch nicht einmal vollständig, denn positive Beschäftigungswirkungen werden schließlich auch noch versprochen. Das ist Grund genug, die geplanten Reformmaßnahmen - insbesondere dessen Kernstück, den weiterentwickelten Zusatzbeitrag - einer genaueren Analyse zu unterziehen. Weil diese die Einhaltung der vollmundigen Reformversprechen allerdings keineswegs bestätigen kann, wird anschließend der Frage nachgegangen, welche gezielten Reformmaßnahmen stattdessen ergriffen werden könnten, damit die GKV-Finanzierung tatsächlich gerechter und stabiler wird.

\section{Stefan Greß, Klaus Jacobs, Sabine Schulze}

\section{Geburt eines Reformkonzepts}

Die erste programmatische Aussage der schwarz-gelben Bundesregierung zur künftigen Finanzierung der gesetzlichen Krankenversicherung (GKV) findet sich naturgemäß im Koalitionsvertrag vom 26.10.2009: „Langfristig wird das bestehende Ausgleichssystem überführt in eine Ordnung mit mehr Beitragsautonomie, regionalen Differenzierungsmöglichkeiten und einkommensunabhängigen Arbeitnehmerbeiträgen, die sozial ausgeglichen werden. Weil wir eine weitgehende Entkoppelung der Gesundheitskosten von den Lohnzusatzkosten wollen, bleibt der Arbeitgeberanteil fest“ (CDU/CSU/FDP 2009: 86). Diese Sätze wurden monatelang zitiert und interpretiert - auch von den Koalitionspartnern in teilweise sehr unterschiedlicher Auslegung -, denn es geschah zunächst weiter nichts. Abgesehen davon, dass sich die Finanzlage der GKV immer weiter verschlechterte, die ersten Krankenkassen im Frühjahr 2010 Zusatzbeiträge erheben mussten und für 2011 ein GKV-Defizit in zweistelliger Milliardenhöhe vorausgesagt wurde.

Prof. Dr. Stefan Greß, Leiter des Fachgebietes Versorgungsforschung und Gesundheitsökonomie an der Hochschule Fulda, Dr. Klaus Jacobs und Dipl.-Volkswirtin Sabine Schulze, Wissenschaftliches Institut der AOK (WIdO) in Berlin
Anfang Juni 2010 trat dann endlich Bundesgesundheitsminister Philipp Rösler mit einem Reformvorschlag an die Öffentlichkeit - dem „Modell einer Gesundheitsprämie mit sozial gestaffelten Beiträgen“ (BMG 2010a) -, der jedoch auf heftige Ablehnung beim bayerischen Koalitionspartner stieß und nur ganze zwei Tage Bestand hatte.

Anfang Juli 2010 einigte sich dann die Bundesregierung auf ein Eckpunkte-Papier mit dem programmatischen Titel „Für ein gerechtes, soziales, stabiles, wettbewerbliches und transparentes Gesundheitssystem" (Bundesregierung 2010). Die darin enthaltenen Maßnahmen sehen kurzfristige Einsparungen und Ausgabenbegrenzungen von 3,5 Mrd. Euro für 2011 bzw. 4,0 Mrd. Euro für 2012 vor, eine zum Jahresbeginn 2011 wirksame Erhöhung des GKVBeitragssatzes von 14,9 auf 15,5 Prozent (bei 8,2 Prozent Versicherten- und 7,3 Prozent Arbeitgeberanteil), der auf diesem Niveau festgeschrieben werden soll, sowie zur Finanzierung der vom Gesundheitsfonds nicht gedeckten Ausgaben einkommensunabhängige kassenindividuelle Zusatzbeiträge mit einem steuerfinanzierten Sozialausgleich vor - wobei allerdings gleich eingeschränkt wurde: „Steuererhöhungen sind hierfür nicht erforderlich."

Bei der Einschätzung des Stellenwerts der einzelnen Reformmaßnahmen gingen die Ansichten der Koalitionspartner allerdings schon bald wieder auseinander - insbesondere im Hinblick auf den kassenindividuellen Zusatzbeitrag. Während einerseits Gesundheitsminister 
Philipp Rösler (in einem am 09.07.2010 an die Mitglieder der Koalitionsfraktionen im Bundestag versandten Argumentionspapier) für das Reformkonzept damit warb, dass „die Weiterentwicklung der Zusatzbeiträge (...) die Voraussetzung (schafft), langfristig die Finanzierung des Gesundheitssystems zu sichern“ (BMG 2010b), äußerte sich CSU-Chef Horst Seehofer nur einen Tag später deutlich zurückhaltender: „Ich kann immer nur schmunzeln, wenn ich wieder tapfere Politikerinnen und Politiker höre, die dann sagen, jetzt haben wir die langfristige Finanzierung gefunden und die wird auf Dauer halten. - Pustekuchen“ (Interview in Deutschlandradio Kultur am 10.07.2010).

Aber unabhängig davon, wie es um die politische Realisierbarkeit und die Dauer der Tragfähigkeit des Instruments Zusatzbeiträge tatsächlich bestellt sein wird - im Interesse der Beitragszahler und aller Versicherten der GKV muss sichergestellt werden, dass dieses Instrument von Beginn an den hohen Ansprüchen entspricht, die die Bundesregierung selbst erhebt.

\section{Beurteilung des schwarz-gelben Reformkonzepts}

In diesem Abschnitt soll das von der Bundesregierung geplante neue Finanzierungskonzept der GKV nach fünf Kriterien untersucht werden: Verteilungsgerechtigkeit, Stabilität und Nachhaltigkeit, Wettbewerbskompatibilität, Beschäftigungswirkungen und Praktikabilität.

\subsection{Verteilungsgerechtigkeit}

Von der Bundesregierung selbst in den Vordergrund gestellt werden die Kriterien „gerecht“ und „sozial“. Wahrscheinlich ist dieses Verhalten von der verständlichen Absicht - wohl besser: Hoffnung - geleitet, dass man eine Regelung nur oft und laut genug als gerecht und sozial bezeichnen muss, damit sie von der Bevölkerung schließlich auch so wahrgenommen wird. Allerdings funktioniert das keineswegs immer, wie zuletzt bereits die Rezeption des als „Zukunftsprogramm“ titulierten Sparpakets gezeigt hat, das auch nicht bereits dadurch „ausgewogen“ wird, dass es von seinen Urhebern ständig so bezeichnet wird. Das wurde besonders deutlich, als Vertreter der CDU-Mittelstandsvereinigung beklagten, dass die Besserverdienenden gar keinen Sparbeitrag leisten würden.

Ähnlich steht es um die aktuellen Pläne zur GKV-Finanzierungsreform: Sie sind nicht „gerecht" und „sozial" und werden es auch durch ständige Wiederholung entsprechender Behauptungen nicht. Allerdings sollte man bei einer differenzierten Analyse nicht nur danach unterscheiden, welcher Art und Ausprägung bestimmte Ungerechtigkeiten im künftigen System der Beitragsgestaltung sind; es gilt auch zu konstatieren, dass ein Großteil dieser Ungerechtigkeiten schon im bestehenden System existiert und durch die geplante Reform insoweit „nur“ noch ein Stück vergrößert wird. Das kann aber weder die Betroffenen trösten noch die Verantwortlichen entlasten.
Letztere nämlich sind angetreten mit großen Ansprüchen, die sie wieder und wieder in der Öffentlichkeit erhoben haben - etwa Philipp Rösler noch kurz vor der Vorstellung des Reformkonzepts (am 05.06.2010) im Deutschlandfunk: „Die unteren Einkommen dürfen nicht stärker belastet werden, und breitere Schultern müssen eben mehr tragen als schmalere."

Genau das geschieht aber nicht, sondern das genaue Gegenteil: Durch die Anhebung des allgemeinen Beitragssatzes werden zunächst alle Einkommen gleichmäßig höher belastet - allerdings nur die beitragspflichtigen Einkommen bis zur Beitragsbemessungsgrenze. Einkommen aus Vermögen oder aus Vermietung und Verpachtung werden auch künftig nicht berücksichtigt und Arbeitsentgelte jenseits der Beitragsbemessungsgrenze von aktuell 3.750 Euro ebenfalls nicht. Das heißt, dass bereits die Anhebung des Beitragssatzes von derzeit 14,9 auf künftig 15,5 Prozent bzw. des Versichertenanteils von 7,9 auf 8,2 Prozent im Ergebnis dazu führt, das die unteren (und ebenfalls die „mittleren“) Einkommen vergleichsweise stärker belastet werden als die höheren. Breitere Schultern tragen also keineswegs mehr, sondern relativ weniger als schmalere.

Diese Belastungswirkungen, die dem in dem Interview artikulierten Gerechtigkeitsanspruch des Gesundheitsministers offensichtlich zuwiderlaufen, sind nicht neu, sondern der derzeitigen Beschränkung der Beitragserhebung auf bestimmte Einkommen bis zur Beitragsbemessungsgrenze geschuldet. Sie bestehen jedoch unverändert fort.

Hinzu kommen mit den in Zukunft obligatorisch pauschal zu erhebenden kassenindividuellen Zusatzbeiträgen nun allerdings neue, zusätzliche Belastungsunterschiede, die sich aus verteilungspolitischer Sicht ebenfalls alles andere als gerecht darstellen. Hieran ändert die als Sozialausgleich bezeichnete Begrenzung der Zusatzbeitrags-Belastung auf maximal zwei Prozent des beitragspflichtigen Einkommens nichts. Schließlich bedeutet bis zu dieser Grenze ein pauschaler Zusatzbeitrag eine umso höhere prozentuale Belastung, je niedriger das Einkommen liegt. Die Bundesregierung rechnet auf der Grundlage von Schätzungen des Bundesversicherungsamtes damit, dass ab 2012 GKV-weit Zusatzbeiträge erhoben werden müssen, weil die Mittel des Gesundheitsfonds zur Deckung der gesamten GKV-Ausgaben nicht mehr ausreichen. 2012 wird ein durchschnittlicher Zusatzbeitrag von 8 Euro erwartet, 2013 von 12 Euro und 2014 von 16 Euro. Das heißt, dass der auf eine maximale Zusatzbeitrags-Begrenzung von zwei Prozent ausgerichtete Sozialausgleich bei beitragspflichtigen Einkommen bis zu 400 (2012), 600 (2013) bzw. 800 Euro (2014) „greift“. Bezieher von Einkommen bis zu dieser Höhe werden jeweils mit den vollen zwei Prozent belastet. Gleichzeitig fällt die Belastung durch den Zusatzbeitrag für Besserverdienende prozentual geringer aus, und zwar umso geringer, je höher das Einkommen.

Diese Wirkung veranschaulicht Tabelle 1. Die Ergebnisse der Tabelle bedürfen noch einer besonderen Erläuterung für den Einkommensbereich bis 800 Euro. Im Rahmen der „Hartz-Gesetzgebung“ - hier konkret: der seit April 2003 geltenden „Hartz II-Regelungen“ - sind die sogenannten 
geringfügigen Beschäftigungsverhältnisse neu geregelt worden. Seither gilt: Wer als Arbeitnehmer 400 Euro oder weniger verdient („Mini-Job“), bezahlt selbst keinen eigenständigen Krankenversicherungsbeitrag - dieser wird im Rahmen einer Pauschalabgabe ausschließlich von den Arbeitgebern getragen. In der sogenannten „Gleitzone“ von 401 bis 800 Euro („Midi-Job“) zahlt der Arbeitgeber den vollen Beitragssatz - also künftig 7,3 Prozent - an die GKV, der Arbeitnehmer jedoch einen reduzierten Beitrag; dieser kommt technisch dadurch zustande, dass nicht das tatsächliche Arbeitsentgelt (also z.B. 600 Euro), sondern ein demgegenüber vermindertes fiktives Entgelt (in diesem Fall 551,70 Euro) als Beitragsbemessungsbasis herangezogen wird. Der Abstand dieses fiktiven Entgelts, auf das der Versicherten-Beitragsatz von künftig 8,2 Prozent bezogen wird, zum tatsächlichen Bruttoarbeitsentgelt nimmt mit wachsendem Einkommen ab, bis am oberen Ende der „Gleitzone“ bei einem Entgelt von 800 Euro keine entsprechende Differenz mehr besteht, der Versichertenbeitrag also - wie bei allen höheren Einkommen bis zur Beitragsbemessungsgrenze - auf der Grundlage des tatsächlichen Bruttoarbeitsentgelts erhoben wird.

Auf diese Regelung wird bei der Beurteilung der geplanten Reformmaßnahmen im Hinblick auf ihre Beschäftigungswirkungen noch einzugehen sein - in der hier zunächst im Vordergrund stehenden verteilungspolitischen Sicht hat sie zur Folge, dass bei Arbeitnehmern mit geringfügiger Beschäftigung bis zu 800 Euro eine unterproportionale Belastung durch die einkommensabhängigen Krankenversicherungsbeiträge zum Tragen kommt. In Be- zug auf den Zusatzbeitrag ist derzeit noch unklar, auf welches Einkommen die Zwei-Prozent-Überforderungsgrenze künftig bezogen wird: auf die fiktiven - gegenüber dem Arbeitsentgelt reduzierten - beitragspflichtigen Einnahmen (wie in Tabelle 1 unterstellt) oder auf das tatsächliche Bruttoarbeitsentgelt. So oder so werden die betroffenen Arbeitnehmer mit Einkommen aus geringfügiger Beschäftigung durch die pauschalen Zusatzbeiträge vergleichsweise kräftig zur Kasse gebeten.

Für andere Versicherte mit beitragspflichtigen Einnahmen in diesem Einkommensbereich, also insbesondere für Kleinrentner, aber auch z.B. für Auszubildende, gelten die beschriebenen „Gleitzonen-Regelungen“ ohnehin nicht. Bei ihnen gibt es weder in Bezug auf die einkommensabhängigen Beitragszahlungen, noch beim ZusatzbeitragsSozialausgleich irgendwelche Regelungen, die zu einer Minderung der Beitragsbelastung führen.

Generell gilt, dass der relative Anteil des pauschalen $\mathrm{Zu}$ satzbeitrags am beitragspflichtigen Mitgliedseinkommen mit steigendem Einkommen abnimmt. Bei Einnahmen in Höhe der derzeitigen Beitragsbemessungsgrenze von 3.750 Euro pro Monat bedeutet ein Zusatzbeitrag von 16 Euro nur noch eine prozentuale Belastung von 0,4 Prozent, sodass sich die Gesamtbelastung aus Versichertenbeitrag und Zusatzbeitrag auf 8,6 Prozent summiert. Bei Einkommen oberhalb der Beitragsbemessungsgrenze kommt zusätzlich deren regressive Wirkung zum Tragen, sodass die BeitragsGesamtbelastung bei einem GKV-Mitglied mit 8000 Euro Bruttomonatsentgelt gerade noch 4 Prozent ausmacht.

Tabelle 1: Beitragsbelastung nach Einkommen bei 16 Euro Zusatzbeitrag

\begin{tabular}{|c|c|c|c|c|c|}
\hline $\begin{array}{c}\text { Brutto- } \\
\text { Einkommen }\end{array}$ & $\begin{array}{c}\text { Vers.beitrag } \\
\text { bei } 8,2 \%\end{array}$ & $\begin{array}{c}\text { Sozial- } \\
\text { ausgleich }\end{array}$ & $\begin{array}{l}\text { Zusatzbeitr. } \\
\text { Eigenanteil }\end{array}$ & $\begin{array}{l}\text { Beitrag } \\
\text { gesamt }\end{array}$ & $\begin{array}{l}\text { Gesamt- } \\
\text { Belastung }\end{array}$ \\
\hline \multicolumn{5}{|c|}{ in Euro/Monat } & $\%$ Brutto-EK \\
\hline 450 & 29,97 & 8,69 & 7,31 & 37,28 & 8,3 \\
\hline 450 & 36,90 & 7,00 & 9,00 & 45,90 & 10,2 \\
\hline 600 & 45,24 & 4,97 & 11,03 & 56,27 & 9,4 \\
\hline 600 & 49,20 & 4,00 & 12,00 & 61,20 & 10,2 \\
\hline 800 & 65,60 & 0 & 16,00 & 81,60 & 10,2 \\
\hline 1000 & 82,00 & 0 & 16,00 & 98,00 & 9,8 \\
\hline 1500 & 123,00 & 0 & 16,00 & 139,00 & 9,3 \\
\hline 2500 & 205,00 & 0 & 16,00 & 221,00 & 8,8 \\
\hline 3750 & 307,50 & 0 & 16,00 & 323,50 & 8,6 \\
\hline 5000 & 307,50 & 0 & 16,00 & 323,50 & 6,5 \\
\hline 6500 & 307,50 & 0 & 16,00 & 323,50 & 5,0 \\
\hline 8000 & 307,50 & 0 & 16,00 & 323,50 & 4,0 \\
\hline
\end{tabular}

Bis 800 Euro separat:

Arbeitnehmer mit Midi-Job

Sonstige Mitglieder (u.a. Rentner)

Quelle: eigene Berechnungen 
Diese Wirkung der Beitragsbemessungsgrenze ist nicht neu - neu ist, dass es künftig auch schon bei Einkommen unterhalb der Beitragsbemessungsgrenze regressive Belastungswirkungen gibt.

Kombinationen aus schon bekannten und zusätzlichen „verteilungspolitischen Problemfällen“ treten auch in anderer Hinsicht auf - insbesondere als Folge der fehlenden Berücksichtigung von weiteren Einkommensarten bzw. des Haushaltsbezugs, die sowohl bei der „normalen“ Beitragsbemessung als auch beim künftigen Sozialausgleich für den Zusatzbeitrag zum Tragen kommen. Dazu sind in Tabelle 2 zwei Beispiele dargestellt:

- Zum einen geht es um die unterschiedliche Beitragsbelastung von zwei Ehepaaren, die jeweils über ein gemeinsames Monatseinkommen aus unselbständiger Arbeit von 7.500 Euro verfügen, das jedoch unterschiedlich auf die beiden Partner verteilt ist. Bei einem unterstellten Zusatzbeitrag von 16 Euro beträgt die Gesamtbelastung des ersten Ehepaares mit einer Gleichverteilung der Einkommen 8,6 Prozent. Das gleich hohe Gesamteinkommen des zweiten Ehepaares mit ungleicher
Verteilung der Arbeitsentgelte wird dagegen nur mit 5 Prozent belastet, einschließlich eines steuerfinanzierten Sozialausgleichs von 7,45 Euro im Monat.

- Zum anderen handelt es sich um zwei Rentner mit gleich hohen monatlichen Gesamteinkommen von jeweils 1.850 Euro aus unterschiedlichen Quellen. Beim ersten Rentner, der ausschließlich eine gesetzliche Altersrente bezieht, beläuft sich die Gesamtbelastung aus Versichertenbeitrag und einem unterstellten Zusatzbeitrag von 16 Euro auf 9,1 Prozent. Beim zweiten Rentner, der seinen Lebensunterhalt neben einer kleinen Rente vor allem aus Mieteinkünften bestreitet, beträgt die Gesamtbelastung dagegen lediglich 3,6 Prozent. Dieser Renter erhält sogar einen steuerfinanzierten Sozialausgleich von 3 Euro im Monat.

Gewiss ist der Anteil, den die geplante Ausgestaltung des Sozialausgleichs zur Vergrößerung der bei den beiden Beispielfällen konstatierten Belastungsunterschiede beiträgt, relativ gering. Doch geht es hier um eine Reform, die von ihren Urhebern nicht nur als gerecht, sondern zugleich als Strukturreform mit Zukunftsperspektive bezeichnet

Tabelle 2: Zwei Beispiele für Belastungsunterschiede bei 16 Euro Zusatzbeitrag

\begin{tabular}{|c|c|c|c|c|c|c|}
\hline \multicolumn{6}{|c|}{ Beispiel I: zwei Ehepaare mit gleich hohem Gesamteinkommen } & \multirow[b]{2}{*}{$\begin{array}{l}\text { Gesamt- } \\
\text { belastung }\end{array}$} \\
\hline & $\begin{array}{l}\text { Brutto-Ein- } \\
\text { kommen }\end{array}$ & $\begin{array}{c}\text { Vers.beitrag } \\
\text { bei } 8,2 \%\end{array}$ & $\begin{array}{c}\text { Sozial- } \\
\text { ausgleich }\end{array}$ & $\begin{array}{l}\text { Zusatzbeitr. } \\
\text { Eigenanteil }\end{array}$ & $\begin{array}{l}\text { Beitrag } \\
\text { gesamt }\end{array}$ & \\
\hline & \multicolumn{5}{|c|}{ in Euro/Monat } & $\begin{array}{l}\text { \% v. Brutto- } \\
\text { Einkommen }\end{array}$ \\
\hline \multicolumn{7}{|c|}{ Paar 1: gleich hohe Einkommen } \\
\hline Partner 1 & 3750 & 307,50 & 0,00 & 16,00 & & \\
\hline Partner 2 & 3750 & 307,50 & 0,00 & 16,00 & & \\
\hline Paar 1 & 7500 & 615,00 & 0,00 & 32,00 & 647,00 & 8,6 \\
\hline \multicolumn{7}{|c|}{ Paar 2: unterschiedlich hohe Einkommen } \\
\hline Partner 1 & 7000 & 307,50 & 0,00 & 16,00 & & \\
\hline Partner 2 & 500 & 35,06 & 7,45 & 8,55 & & \\
\hline Paar 2 & 7500 & 342,56 & 7,45 & 24,55 & 374,56 & 5,0 \\
\hline \multicolumn{7}{|c|}{ Beispiel II: zwei Rentner mit gleich hohem Einkommen aus verschiedenen Quellen } \\
\hline \multicolumn{7}{|c|}{ „Nur-Rentner“ 1} \\
\hline Rente & 1850 & 151,70 & 0,00 & 16,00 & 167,70 & 9,1 \\
\hline \multicolumn{7}{|c|}{ Rentner 2: zwei Einkommensquellen } \\
\hline Rente & 650 & 53,30 & 3,00 & 13,00 & & \\
\hline Mieteinkünfte & 1200 & & & & & \\
\hline Gesamt & 1850 & 53,30 & 3,00 & 13,00 & 66,30 & 3,6 \\
\hline
\end{tabular}

Quelle: eigene Berechnungen 


\section{THEMA}

wird. Daran gemessen reicht es nicht, wenn die mit dieser Reform zusätzlich neu geschaffenen verteilungspolitischen Probleme im Vergleich zu den schon vorhandenen und unverändert fortbestehenden Problemen relativ klein ausfallen. Schon gar nicht passt dies mit der eingangs zitierten Ankündigung zusammen, dass breitere Schultern künftig mehr tragen sollen als schmalere.

\subsection{Stabilität und Nachhaltigkeit}

Wie mehrfach gezeigt (zuletzt einmal mehr Wille 2010) und entsprechend lange bekannt, liegt die zentrale Ursache der seit den 1980er Jahren mehr oder weniger unverändert anhaltenden Beitragssatzsteigerungen in der GKV nicht etwa an der demografischen Entwicklung oder Ausgabenzuwächsen, die auch nur im entferntesten die Bezeichnung „Kostenexplosion“ verdienen. Zentrale Ursache der Beitragssatzsteigerungen war und ist vielmehr die Wachstumsschwäche der Finanzierungsbasis. Einerseits machen die GKV-Ausgaben seit über 20 Jahren einen relativ stabilen Anteil von 6 bis 7 Prozent am Bruttoinlandsprodukt aus. Andererseits sind die beitragspflichtigen Einkommen der GKV-Mitglieder aber deutlich hinter dieser Entwicklung zurückgeblieben.

Eine an dieser Problemdiagnose ansetzende Stabilisierung der GKV-Finanzierungsbasis müsste demzufolge bewirken, dass sich die Finanzierungsbasis der GKV in Zukunft wenigstens im selben Maße entwickelt wie die gesamte Wirtschaftskraft bzw. wie das Volkseinkommen. Dazu würde es des Einbezugs derjenigen Teile des Volkseinkommens in die GKV-Finanzierung bedürfen, die hierbei bislang unberücksichtigt bleiben: Arbeitseinkommen jenseits der Beitragsbemessungsgrenze, Einkommen aus anderen Quellen sowie Einkommen von Personen, die als Privatversicherte zumindest bislang überhaupt nicht regelhaft zur Mitfinanzierung herangezogen werden. Hierzu kann es aber nur kommen, wenn die bestehende Finanzierungsbasis in substanziellem Ausmaß ergänzt wird - sei es durch Beitragszahlungen auf bislang beitragsfreie Einkommensarten bzw. Einkommensteile oder durch Steuermittel, die auf unterschiedlichen Wegen zur Stabilisierung der GKV-Finanzen beitragen können (vgl. dazu Abschnitt 3). Wenn jedoch der Gesundheitsminister - sogar mit unverkennbarem Stolz - verkündet, dass zur Finanzierung des Sozialausgleichs auf absehbare Zeit keine Steuererhöhungen erforderlich seien, kann es sich umgekehrt auch nicht um einen nennenswerten Stabilisierungsbeitrag handeln, mit dem die GKV künftig aus Steuermitteln rechnen kann. Wenn diejenigen - Personen wie Einkommen -, die bislang kaum oder gar nicht an der solidarischen Finanzierung beteiligt waren, hierzu künftig verstärkt herangezogen werden sollen, aber zugleich keinerlei Zusatzbelastung spüren sollen, passt das offenkundig schon nach einfacher Logik nicht zusammen.

Für die Stabilität und erst recht für die Nachhaltigkeit der GKV-Finanzierung bedeutet das nichts Gutes, denn am Ende müssen letztlich wieder dieselben Personen mit denselben Einkommen den Großteil der gesamten Finanzie- rungslast schultern. Zusätzliche Stabilität wird das System dadurch jedenfalls nicht bekommen.

\subsection{Wettbewerbskompatibilität}

Die zum Jahresbeginn 2011 geplante Beitragssatzerhöhung von 14,9 auf 15,5 Prozent führt zunächst dazu, dass zumindest kurzfristig wohl kaum weitere Krankenkassen gezwungen sein dürften, einen Zusatzbeitrag von ihren Mitgliedern zu erheben. So erfreulich dies insbesondere für die Mitglieder dieser Kassen ist, so fragwürdig sind gleichwohl die Konsequenzen in Bezug auf den Krankenkassenwettbewerb. Es wird damit nämlich eine Situation fortgesetzt, die seit Einführung des Gesundheitsfonds zum Jahresbeginn 2009 dadurch gekennzeichnet war, dass alle Kassen ihr gesamtes Handeln fast ausschließlich dem Ziel untergeordnet haben, so lange wie möglich keinen Zusatzbeitrag erheben zu müssen. Dass dieses Verhalten sehr wohl begründet war, zeigen die hohen Mitgliederverluste derjenigen Kassen, die mittlerweile Zusatzbeiträge erheben. Laut Frankfurter Allgemeine Zeitung vom 22.07.2010 belaufen sich die Mitgliederverluste bei der DAK auf fünf Prozent und bei der BKK Gesundheit sogar auf bis zu 20 Prozent - beide Kassen verlangen seit Frühjahr 2010 einen Zusatzbeitrag von monatlich 8 Euro.

Das vorrangige Bestreben der Kassen, so lange wie möglich keinen Zusatzbeitrag erheben zu müssen, hat seit Anfang 2009 dazu geführt, alle kurzfristig disponiblen Kassenausgaben soweit wie möglich zurückzufahren. Das hat - bei gleichzeitigem Auslaufen der pauschalen Anschubfinanzierung - auch zu einer erkennbaren Zurückhaltung in Bezug auf Verträge zur integrierten Versorgung geführt, zumindest wenn diese mit anfänglichem Investitionsbedarf verbunden sind, der erst längerfristig über Effizienzsteigerungen in der Versorgung refinanziert werden kann. Nach regelmäßig zweistelligen Zuwachsraten sind die Gesamtausgaben aller Kassen für Leistungen der integrierten Versorgung 2009 im Vergleich zum Vorjahr sogar leicht gesunken. Dies betrifft gerade solche Leistungen, die ausschließlich auf der Grundlage von Selektivverträgen zwischen Krankenkassen und Anbietern von Gesundheitsleistungen zustande kommen und im Hinblick auf qualitäts- und effizienzfördernden Vertragswettbewerb als besonders vielversprechend gelten (Cassel et al. 2006, aktuell auch Monopolkommission 2010). Aufgrund der geplanten Beitragssatzerhöhung und des damit verbundenen Aufschubs der GKV-weiten „Normalität“ kassenindividueller Zusatzbeiträge dürfte der dadurch verursachte vertragswettbewerbliche Attentismus entsprechend fortbestehen. Im Hinblick auf die Realisierung eines GKV-Wettbewerbskonzepts zur Verbesserung von Qualität und Wirtschaftlichkeit der Gesundheitsversorgung, in dem die kassenindividuellen Zusatzbeiträge eine zentrale Rolle spielen sollen, ist die abermalige Verschiebung ihrer flächendeckenden Einführung deshalb grundsätzlich kontraproduktiv.

Positiv an den Reformplänen der Bundesregierung ist dagegen zu bewerten, dass zwei bislang aus der dysfunk- 
tionalen Konstruktion des Zusatzbeitrags resultierende Wettbewerbsprobleme künftig beseitigt werden sollen. Das gilt zunächst und vor allem für die bisherige Überforderungsklausel, die insbesondere Krankenkassen mit vielen einkommensschwachen Mitgliedern - unbeschadet aller Wirtschaftlichkeitsbemühungen - unvermeidlich in die Insolvenz getrieben hätte. Eine solche „Insolvenzfalle“ wäre entstanden, wenn Krankenkassen die Differenz zwischen ihren Ausgaben und den Zuweisungen aus dem Gesundheitsfonds aufgrund der bisherigen 1-ProzentÜberforderungsklausel nicht mehr durch Zusatzbeiträge ihrer Mitglieder hätten schließen können (vgl. Schawo 2007).

Dieses Problem war bereits bekannt, noch ehe diese untaugliche Konstruktion mit dem GKV-Wettbewerbsstärkungsgesetz Anfang 2007 beschlossen wurde. Schließlich waren im Zuge der Gesetzgebung im Herbst 2006 eigens zwei gutachterliche Stellungnahmen von Eckart Fiedler und Bert Rürup eingeholt worden, in denen der Mechanismus der „Insolvenzfalle“ nicht nur nachdrücklich beschrieben, sondern zugleich praktikable Lösungswege aufzeigt wurden. Dass die Politik jetzt endlich handelt und diesen groben Konstruktionsfehler beim Zusatzbeitrag beseitigt, erscheint somit mehr als überfällig. Wenn dabei jedoch ein vermeintlich sachlogischer Zusammenhang mit der gleichzeitig beabsichtigten Anhebung der Überforderungsgrenze von 1 auf 2 Prozent der beitragspflichtigen Einnahmen konstruiert wird, kann dies nur als unseriös bezeichnet werden. Die Aussage von Gesundheitsminister Rösler - „Ein System, das mit zwei Prozent funktioniert, ist besser als ein System mit einem Prozent, das schon im nächsten Jahr vor die Wand fährt. " (Interview in der Hannoverschen Allgemeinen Zeitung vom 17.07.2010) blendet aus, dass ein funktionierendes Ausgleichssystem auch unter Fortbestand der bisherigen 1-Prozent-Überforderungsgrenze einfach zu installieren gewesen wäre. Bei der jetzt vorgesehenen Konstruktion des Sozialausgleichs würde das allerdings von Anfang an einen höheren Steuerzuschuss erfordern.

Das zweite Problem der bisherigen Zusatzbeitragsregelung aus wettbewerblicher Sicht resultierte daraus, dass sich der Sozialausgleich in Gestalt der 1-ProzentÜberforderungsklausel auf den tatsächlich erhobenen Zusatzbeitrag einer Kasse bezog. Das hätte für immer mehr einkommensschwächere Mitglieder zur Folge gehabt, dass der Zusatzbeitrag für sie keine Rolle mehr als Wettbewerbsparameter gespielt hätte, weil die maximale Belastung von einem Prozent der beitragspflichtigen Einnahmen praktisch bei jeder Kasse mit irgendeinem Zusatzbeitrag erreicht worden wäre. Damit hätte der Zusatzbeitrag die ihm zugeschriebene Signalfunktion im Krankenkassenwettbewerb für immer mehr GKV-Mitglieder eingebüßt. Um diese Wirkung zu vermeiden, soll sich der Sozialausgleich künftig auf den GKV-durchschnittlichen Zusatzbeitrag beziehen. Damit bleibt es auch für Geringverdiener lohnend, in eine günstigere Kasse zu wechseln, weil der auf Basis des durchschnittlichen Zusatzbeitrags ermittelte Ausgleich in jedem Fall erhalten bleibt. In einer Kasse mit überdurchschnittlich hohem Zusatzbeitrag muss dagegen die Diffe- renz zum GKV-Durchschnitt in jedem Fall vom Versicherten getragen werden. Aus wettbewerbsökonomischer Sicht ist diese Regelung zu begrüßen; ob es dagegen $u$. U. zu rechtlichen Problemen führt, wenn z.B. ein Mitglied einer Kasse ohne Zusatzbeitrag dennoch einen steuerfinanzierten „Ausgleich“ erhält, steht auf einem anderen Blatt.

Diese in wettbewerblicher Sicht sinnvolle Regelung setzt allerdings zwingend voraus, dass der Zusatzbeitrag auch tatsächlich ein unverzerrtes Preissignal aussendet. Hieran bestehen jedoch durchaus einige Zweifel, von denen hier vier kurz genannt seien:

- Der im Hinblick auf das ausgabenrelevante Krankheitspanorama unvollständige morbiditätsorientierte Risikostrukturausgleich führt im Ergebnis zu einer Benachteiligung von Krankenkassen mit überdurchschnittlich vielen Versicherten mit „falschen“ Krankheiten, weil diese im Risikostrukturausgleich infolge der gesetzlichen Beschränkung auf maximal 80 Krankheiten nicht in Form eigenständiger Risikozuschläge berücksichtigt werden (vgl. Jacobs 2008).

- Krankenkassen mit vielen einkommensschwachen Mitgliedern haben unter sonst gleichen Umständen vergleichsweise höhere Leistungsausgaben zu finanzieren, weil die von den Patienten bei der Inanspruchnahme von Versorgungsleistungen zu entrichtenden Zuzahlungen durch Härtefall-Regelungen begrenzt sind und die hierdurch entstehenden Zahlungsausfälle von allen Beitragszahlern der jeweiligen Kasse ausgeglichen werden müssen (vgl. Jacobs et al. 2002).

- Krankenkassen mit unterschiedlichem regionalen $\mathrm{Zu}$ schnitt ihres Tätigkeitsbereichs haben entsprechend unterschiedliche Zusatzbeitragsregionen, was speziell bei Regionalkassen (oder überregionalen Kassen mit starker regionaler Versichertenkonzentration) in unter- oder überdurchschnittlich ausgabenintensiven Regionen zu zusatzbeitragsrelevanten Wettbewerbsvor- oder -nachteilen führen kann (vgl. Wasem/Greß/Jacobs 2007).

- Krankenkassen mit unterschiedlichen Anteilen an säumigen Zusatzbeitrags-Zahlern müssen ggf. allein aus diesem Grund unterschiedlich hohe Zusatzbeiträge erheben (vgl. hierzu gesondert Abschnitt 2.5).

Diese vier Problemfelder signalisieren weiteren Handlungsbedarf des Gesetzgebers in Bezug auf die Weiterentwicklung der GKV-Wettbewerbsordnung als Voraussetzung für einen möglichst unverzerrten Krankenkassenwettbewerb. Allerdings stellt eine noch so vollständige Ordnung des Krankenkassenwettbewerbs immer nur eine notwendige, aber noch lange keine hinreichende Funktionsbedingung dafür dar, dass produktiver Kassenwettbewerb im Interesse von mehr Qualität, Wirtschaftlichkeit und Präferenzgerechtigkeit der Gesundheitsversorgung auch tatsächlich zustande kommt. Hierzu bedarf es gegenüber dem Status quo nach wie vor deutlich erweiterter wettbewerblicher Handlungsspielräume der Kassen, insbesondere im Vertragsbereich (Cassel et al. 2008, Monopolkommission 2010); andernfalls droht ein noch so intensivierter Kassenwettbewerb auch künftig weitgehend ins Leere zu 


\section{THEMA}

laufen, und das ihm grundsätzlich innewohnende Steuerungspotenzial würde weiterhin großenteils verschenkt.

\subsection{Beschäftigungswirkungen}

Schon im Koalitionsvertrag hatte die Bundesregierung angekündigt: „Weil wir eine weitgehende Entkoppelung der Gesundheitskosten von den Lohnzusatzkosten wollen, bleibt der Arbeitgeberanteil fest" (CDU/CSU/FDP 2009: 86). Diese Ankündigung wird nun umgesetzt: „Der Arbeitgeberbeitrag wird auf der Höhe von 7,3 Prozent festgeschrieben. Damit werden die Gesundheitskosten von den Arbeitskosten für die Zukunft entkoppelt. Unvermeidbare Ausgabensteigerungen werden durch Zusatzbeiträge der Versicherten finanziert" (Bundesregierung 2010).

Ganz abgesehen davon, was nun eigentlich wovon „entkoppelt“ werden soll - die Gesundheitskosten von den Arbeitskosten, wie behauptet, oder nicht doch eher umgekehrt die Arbeitskosten von den Gesundheitskosten? -, stimmt diese Aussage insoweit auch nur eingeschränkt, als über den prozentualen Arbeitgeberbeitrag auch in Zukunft steigende GKV-Ausgaben von den Arbeitgebern mitfinanziert werden - allerdings nur in dem Maße, wie sich die beitragspflichtigen Arbeitsentgelte entwickeln. Allein bei der Finanzierung der über diese Entwicklung hinausgehenden Ausgabenzuwächse der GKV - wie sie in der Vergangenheit allerdings nicht zuletzt wegen der schon angesprochenen Wachstumsschwäche der GKV-Finanzierungsbasis mehr oder weniger die Regel waren (siehe Abschnitt 2.2) - sind die Arbeitgeber in Zukunft nicht mehr beteiligt. (Auf die Frage, welche Konsequenzen sich hieraus für die künftigen Beteiligung der Arbeitgeber an der Selbstverwaltung der Krankenkassen ergeben - die autonom von der Selbstverwaltung der Kassen festgelegten Zusatzbeiträge werden schließlich allein von den Versicherten entrichtet -, sei hier nur am Rande hingewiesen.)

Die Arbeitskosten- (oder Lohnzusatz- bzw. Lohnnebenkosten-) Argumentation ist seit Langem bekannt und trotz wiederholter grundlegender Relativierung und Kritik (z.B. Häussler/Ecker/Schneider 2006, Reiners 2009: 41-52) offensichtlich durch nichts zu erschüttern: Danach beeinträchtigen steigende Lohnzusatz- bzw. Lohnnebenkosten, die insbesondere auf steigende Sozialversicherungsbeiträge zurückgeführt werden, die Wachstums- und Beschäftigungschancen am Wirtschaftsstandort Deutschland.

Somit überrascht es wenig, dass im Hinblick auf die Beschäftigungswirkungen die Arbeitskostensicht der Arbeitgeber bei der geplanten Finanzierungsreform eine zentrale Rolle spielt. Allerdings ist es schon einigermaßen überraschend, dass die Arbeitsangebotsperspektive der Arbeitnehmer offenbar vollständig ausgeblendet wird. Das ist deshalb überraschend, weil die entsprechende Argumentation - Stichwort: Lohnabstandsgebot: Arbeit muss sich (wieder) lohnen - vielen Vertretern der Bundesregierung ansonsten ja keineswegs fremd ist.

Was aber geschieht nun beim Zusatzbeitrag? Wie Tabelle 1 beispielhaft zeigt, werden gerade die Bezieher niedriger
Arbeitseinkommen durch den pauschalen Zusatzbeitrag überproportional belastet, denn die Zwei-Prozent-Belastungsgrenze ist bei ihnen vergleichsweise schnell erreicht. Gerade bei Geringverdienern stellt sich aber nach der gängigen „Lohnabstandsgebot-Argumentation“ die Frage, ob es sich für sie überhaupt noch lohnt, sozialversicherungspflichtig zu arbeiten oder lieber - ggf. in Kombination mit Schwarzarbeit - Sozialleistungen in Anspruch zu nehmen. Gerade die bereits angesprochenen „Midi-Job-Regelungen“ (siehe Abschnitt 2.1) zeigen ja, dass diesem Aspekt bei der Hartz II-Gesetzgebung - im Übrigen auf Betreiben der unionsgeführten Bundesländer - spezielle Aufmerksamkeit gewidmet wurde. So wird bei einem „Midi-Jobber“ mit einem Bruttomonatsentgelt von 600 Euro die Beitragsbemessungsgrundlage für den Versichertenbeitrag eigens auf 551,70 Euro und damit der Versichertenbeitrag - beim künftigen Satz von 8,2 Prozent - von 49,20 auf 45,24 Euro reduziert, um die Anreize zur Aufnahme einer entsprechenden Beschäftigung zu erhöhen. Dem hiermit verbundenen „mehr Netto vom Brutto“ in Höhe von rund 4 Euro wird allerdings künftig - bei einem GKV-durchschnittlichen Zusatzbeitrag von 16 Euro, wie er derzeit für 2014 erwartet wird - ein Eigenanteil beim Zusatzbeitrag von 11 bzw. 12 Euro gegenüberstehen (je nachdem, ob der 2-Prozent-Sozialausgleich auf das fiktiv reduzierte oder das tatsächliche Bruttoentgelt bezogen wird). Wenn die Annahme der Politik bei der Hartz II-Gesetzgebung zutreffend war, dass die Erhöhung des Nettoentgelts um 4 Euro zu positiven Beschäftigungswirkungen im Sinne erhöhter Arbeitsangebotsanreize führt, kann eine künftig deutlich stärkere Minderung des Nettoentgelts durch den nach Sozialausgleich verbleibenden Eigenanteil am Zusatzbeitrag wohl kaum ohne negative Beschäftigungswirkungen bleiben. Nach derselben Argumentation ist demzufolge damit zu rechnen, dass Anreize zur Aufnahme gering entlohnter Beschäftigungsverhältnisse gemindert und Anreize zu Schwarzarbeit erhöht werden.

Dass Gesundheitsminister Rösler hierin dennoch keinen Verstoß gegen das Wahlversprechen seiner Partei „mehr Netto vom Brutto“ erkennen kann, hat er kürzlich in einem Zeitungsinterview dankenswerterweise klar ausgesprochen: „Damit war eindeutig das Steuersystem gemeint" (Welt am Sonntag vom 11.07.2010). Wer keine Beiträge zur gesetzlichen Krankenversicherung bezahlt, kann mit dieser Klarstellung gewiss gut leben. Den MidiJobber - und mit ihm zugleich seinen Arbeitgeber - dürfte sie allerdings nur wenig trösten.

\subsection{Praktikabilität}

Der steuerfinanzierte Sozialausgleich, der eine Überforderung der GKV-Mitglieder durch den Zusatzbeitrag verhindern soll, soll laut Bundesregierung unbürokratisch und „automatisch“ - das heißt insbesondere ohne Antragstellung - beim Arbeitgeber bzw. Rentenversicherungsträger umgesetzt werden. Der Präsident der Deutschen Rentenversicherung, Herbert Rische, hat sich dazu bereits öffentlich geäußert und kritisiert, dass die Rentenversicherung zum „Ersatzfinanzamt“ gemacht werde. Originalton 
Rische: „Automatisch und auf Knopfdruck wird nichts funktionieren. Es muss ja zum Beispiel geprüft werden, ob ein Rentner zusätzlich eine Betriebsrente oder eine Beamtenpension bezieht. Oder ob er nebenher arbeiten geht. Diese Daten liegen uns nicht vor. Wenn wir das leisten sollen, bekommen wir sicher ein Problem“ (Tagesspiegel vom 12.07.2010).

Wenn der steuerfinanzierte Sozialausgleich nicht vollends zur Farce werden soll, weil etwa ein Kleinrentner in den Genuss einer steuerfinanzierten Ausgleichszahlung kommt, obwohl er über eine stattliche Betriebsrente oder Beamtenpension verfügt, geht an der Zusammenführung der entsprechenden Einkommensinformationen kein Weg vorbei (zu einer verfassungsrechtlichen Beurteilung dieser Frage vgl. Ebsen 2010). Sollte das nicht ohne Beteiligung der Finanzämter gehen, stünde damit aber vermutlich die von der Bundesregierung angestrebte Zustimmungsfreiheit des Gesetzesvorhabens im Bundesrat auf dem Spiel.

Ein weiteres Problem kommt bei den Beschäftigten hinzu, bei denen der „automatische“ Sozialausgleich durch die Arbeitgeber erfolgen soll. „Die Regelung ist für die Arbeitgeber leicht handhabbar, da die Lohnabrechnung heute schon EDV-gestützt erfolgt" - so die Bundesregierung (2010) in ihrem Eckpunkte-Papier. Natürlich gilt zunächst das von Herbert Rische für Rentner angesprochene Problem der Zusammenführung von Informationen verschiedener Einkommen genauso für Arbeitnehmer. Fände keine entsprechende Zusammenführung statt, erhielte z.B. ein Arbeitnehmer mit zwei 450 Euro-Jobs bei einem durchschnittlichen Zusatzbeitrag von 16 Euro eine zweimalige Erhöhung seines Nettoentgelts, die in der Summe den mittleren Zusatzbeitrag überstiege (vgl. Tabelle 1) - also ein typischer Fall von Überkompensation, die sich bei Aufnahme eines dritten und vierten Jobs noch weiter vergrößern würde. Nun kann man sich bei der Rentenversicherung als „hoheitlicher Instanz" noch vorstellen, dass dort die Funktion eines „Ersatzfinanzamts“ wahrgenommen wird, wenn der Gesetzgeber das so verlangt. Aber beim Arbeitgeber? Unterliegen Informationen über Einkommen aus anderen Beschäftigungsverhältnissen - vielleicht sogar schon Informationen die Existenz anderer Beschäftigungsverhältnisse - nicht dem Recht auf informationelle Selbstbestimmung (dessen Preisgabe in diesem Fall überdies mit einer höheren Zusatzbeitragsbelastung verbunden, also auch ökonomisch nicht sonderlich rational wäre)?

Man darf gespannt sein, welche konkreten Antworten auf diese praktischen Umsetzungsfragen gefunden werden - neben den genannten etwa auch auf die Frage, wer den Sozialausgleich eigentlich bei freiwillig versicherten Selbständigen durchführen soll, die weder Arbeitgeber noch Rentenversicherungsträger haben. Nach der parlamentarischen Sommerpause soll ein erster Gesetzentwurf vorgelegt werden, der die Fragen beantworten muss.

Ein weiteres praktisches Problem kommt beim Instrument Zusatzbeiträge aber noch hinzu, nämlich die absehbaren Zahlungsausfälle. Dem Präsidenten des Bundesversicherungsamts, Maximilian Gaßner, zufolge „(werden) die bislang von einigen Kassen erhobenen Zusatzbeiträge
(...) von einem Drittel der betroffenen Versicherten nicht bezahlt" (zitiert nach Ärzte Zeitung vom 17.06.2010). Diese Entwicklung ist in mehrerlei Hinsicht problematisch.

Erstens werden Krankenkassen zukünftig in steigendem Ausmaß zu Inkassostellen, um säumige Versicherte zur Beitragszahlung zu bringen. Abgesehen davon, dass den Krankenkassen hierzu nur beschränkte Sanktionsmöglichkeiten zur Verfügung stehen, erhöht dies massiv die mit dem Instrument Zusatzbeitrag verbundenen Bürokratiekosten. Realistischerweise müssen die Krankenkassen daher zur Deckung von Zahlungsausfällen und Bürokratiekosten einen Zusatzbeitrag erheben, der deutlich über dem rechnerisch ausgabendeckenden Zusatzbeitrag liegt.

Zweitens entstehen durch die Zahlungsausfälle Wettbewerbsverzerrungen (vgl. Abschnitt 2.3), wenn - und hiervon ist auszugehen - der Anteil säumiger Versicherter ungleich zwischen den Krankenkassen verteilt ist. Es ist damit zu rechnen, dass vor allem Krankenkassen mit einem hohen Anteil Versicherter mit niedrigem Einkommen die, wie in Abschnitt 2.1 gezeigt, durch die Zusatzbeiträge überproportional stark belastet werden - einen Wettbewerbsnachteil erleiden werden.

Drittens wird sich das Problem verschärfen, wenn der Zusatzbeitrag in Zukunft einen immer größeren Anteil der GKV-Ausgaben finanzieren muss. Die Erfahrungen aus den Niederlanden zeigen, dass der Anteil säumiger Versicherter nach dem Anstieg der pauschal von den Versicherten zu erhebenden Pauschalbeiträge massiv angestiegen ist. Konsequenterweise haben die Autoren der wissenschaftlichen Evaluation der niederländischen Krankenversicherungsreform eine deutliche Senkung des durch Pauschalbeiträge zu finanzierenden Ausgabenanteils empfohlen (van de Ven et al. 2009).

\section{Reformalternativen}

Aus den bisherigen Überlegungen ist deutlich geworden, dass die Pläne der Bundesregierung keine Strukturreform im Sinne einer stabilen, gerechten und praktikablen Finanzierung der gesetzlichen Krankenversicherung darstellen. Die doppelte Beitragserhöhung belastet vor allem die gesetzlich Versicherten mit niedrigen und mittleren Einkommen. Bestehende Nachhaltigkeits- und Gerechtigkeitsdefizite der GKV-Finanzierung bleiben entgegen der Behauptungen der politisch verantwortlichen Akteure bestehen oder werden sogar noch verschärft. Darüber hinaus steigen die Bürokratiekosten durch die Erhebung der Zusatzbeiträge und die Durchführung des Sozialausgleichs, ohne dass der Versichertengemeinschaft durch diesen zusätzlichen Bürokratieaufwand ein erkennbarer Nutzen entstünde.

Am Ende dieses Beitrags sollen daher Überlegungen stehen, wie Kernelemente einer echten Finanzierungsreform jenseits der Regierungspläne aussehen könnten. Es werden zwei Ansätze diskutiert - die zusätzliche Heranziehung von Steuermitteln zur Finanzierung der Ausgaben in der GKV und eine echte Strukturreform der Beitragsfinanzierung. 


\section{THEMA}

\subsection{Reformalternative I: Steuerfinanzierung}

Seit der Implementierung des GKV-Modernisierungsgesetzes (GMG) im Jahr 2004 ist die Bedeutung des Bundeszuschusses zur Finanzierung der gesetzlichen Krankenversicherung deutlich angestiegen. Dieser sowohl quantitative als auch qualitative Bedeutungszuwachs erfolgte jedoch nicht kontinuierlich, sondern ausgesprochen erratisch (vgl. Abbildung 1). Der von der rot-grünen Bundesregierung vorgesehene Ausbau des Bundeszuschusses wurde von der großen Koalition im Rahmen des Haushaltsbegleitgesetzes im Jahr 2006 zunächst gestoppt. Im Rahmen des GKV-Wettbewerbsstärkungsgesetzes (WSG) hat die gleiche große Koalition den Finanzierungsbeitrag des Bundes durch Einzahlungen in den Gesundheitsfonds deutlich ausgebaut. Durch das Konjunkturpaket II stieg der Bundeszuschuss weiter an.

Auch die schwarz-gelbe Regierung nutzt den Bundeszuschuss als probates Mittel zum Ausgleich von Defiziten im Gesundheitsfonds. Durch einen außerordentlichen Bundeszuschuss in Höhe von 3,9 Mrd. Euro im Jahr sollten die Einnahmeausfälle als Folge der Finanz- und Wirtschaftskrise kompensiert werden. Insgesamt werden damit neun Prozent der für das Jahr 2010 geschätzten GKV-Ausgaben (174 Mrd. Euro) aus Steuermitteln finanziert. Im Rahmen des im Juni 2010 beschlossenen Sparpakets wurde darüber hinaus ein weiterer einmaliger Bundeszuschuss in Höhe von 2 Mrd. Euro für das Jahr 2011 beschlossen. Seit Einführung des Bundeszuschusses im Jahr 2004 hat sich die geplante Höhe der Steuerfinanzierung bis Juni 2010 unter drei verschiedenen Regierungen damit insgesamt fünfmal verändert. Viermal haben die jeweiligen Regierungen eine Erhöhung des Bundeszuschusses beschlossen, einmal sollte der Bundeszuschuss zurückgeführt bzw. ganz abgeschafft werden.

Im Vergleich zum Status quo der GKV- Finanzierung weist die Steuerfinanzierung eine Reihe von Vorteilen auf. Die Bemessungsgrundlage sowohl von direkten Steuern wie der Einkommenssteuer als auch von indirekten Steuern wie der Mehrwertsteuer ist deutlich breiter als die Basis der derzeitigen Beitragsfinanzierung. Dies gilt sowohl im Hinblick auf den erfassten Personenkreis als auch im Hinblick auf die zu verbeitragenden bzw. zu versteuernden Einkommensarten. Auf den ersten Blick wirkt die Steuerfinanzierung damit nachhaltiger als die derzeitige Beitragsfinanzierung.

Ein ähnliches Bild ergibt der Vergleich der beiden Finanzierungsarten im Hinblick auf die Gerechtigkeit der Finanzierung. Die Beitragsfinanzierung wirkt jenseits der Beitragsbemessungsgrenze - bei Einführung von Zusatzbeiträgen gilt dies auch für niedrigere Einkommen - regressiv.

Abbildung 1: Bundeszuschuss zur GKV 2003 bis 2011

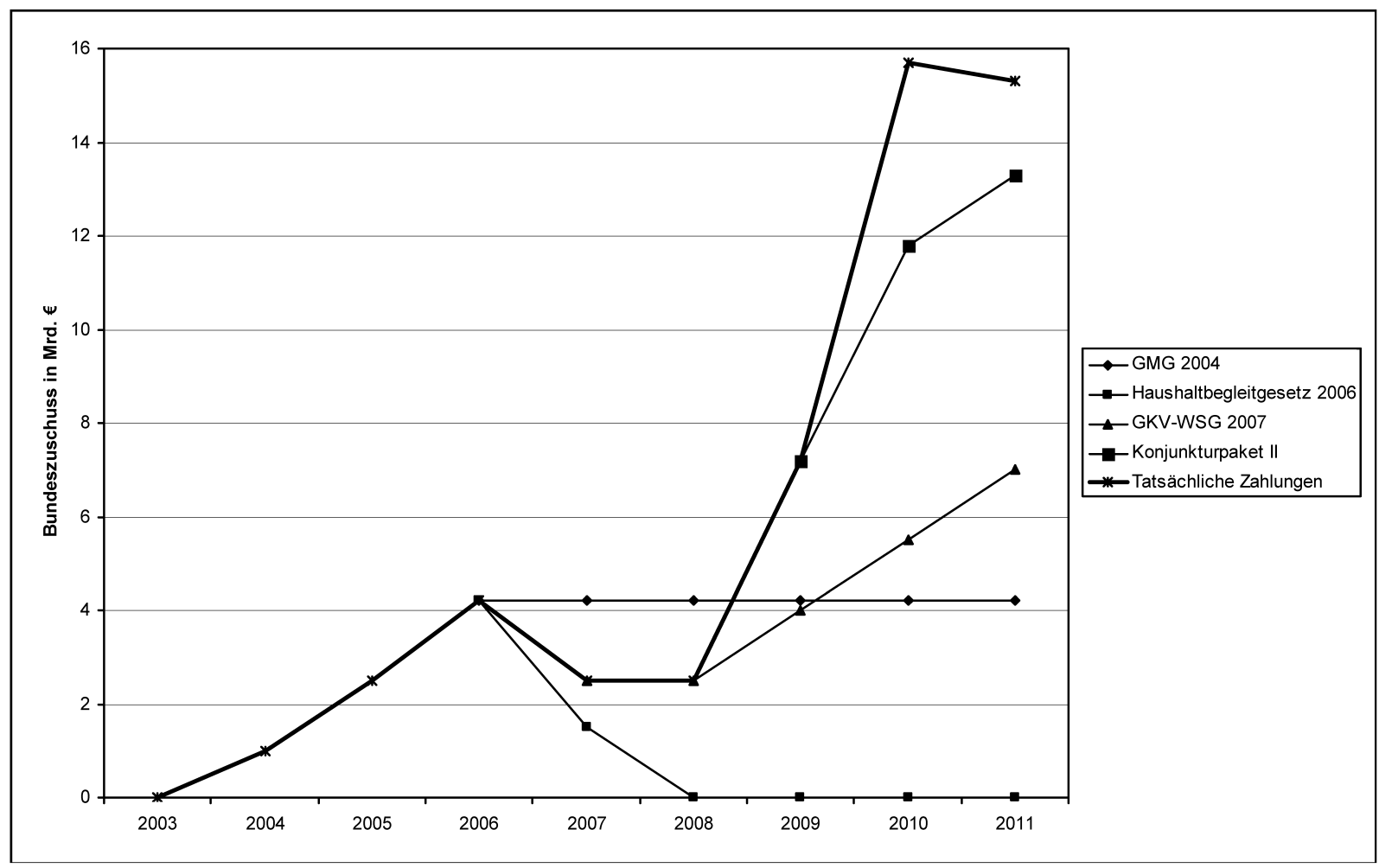

Quelle: Eigene Abbildung 
Die prozentuale Belastung sinkt mit steigendem Einkommen. Die Einkommenssteuer dagegen wirkt bis zum Spitzensteuersatz progressiv. Die prozentuale Belastung steigt mit steigendem Einkommen. Selbst nach Erreichen des Spitzensteuersatzes wirkt die Einkommensteuer proportional - die prozentuale Belastung bleibt bei steigendem Einkommen gleich. Verschiedene Simulationen haben darüber hinaus ergeben, dass durch eine Substitution von Beitrags- durch Steuerfinanzierung moderate Beschäftigungszuwächse erzielt werden können (Meinhardt/Zwiener 2005).

Die genannten Vorteile müssen jedoch relativiert werden. Die Nachhaltigkeit der Finanzierung ist nicht nur von der Bemessungsgrundlage abhängig. Die Erfahrungen etwa bei der Investitionsfinanzierung der Krankenhäuser durch die Bundesländer zeigen, dass die Nachhaltigkeit von steuerfinanzierten Gesundheitsausgaben durch sinkende fiskalische Spielräume akut gefährdet wird. Bisher hat sich diese Erfahrung beim Bundeszuschuss zur GKV nur einmal im Rahmen des Haushaltsbegleitgesetzes 2006 wiederholt. Auch im Rahmen des im Juni 2010 beschlossenen Sparpakets - geprägt von einer Reihe von gravierenden Kürzungen im Bereich Arbeit und Soziales - musste der Bundesgesundheitsminister keine Kürzung seines Etats hinnehmen. Es ist jedoch keinesfalls gesichert, dass diese Entwicklung Bestand hat. Dies gilt insbesondere dann, wenn aus dem Bundeshaushalt nicht nur Leistungsausgaben, sondern auch der Sozialausgleich finanziert werden muss.

Auch die Gerechtigkeitsvorteile auf Seiten der Steuerfinanzierung müssen relativiert werden. Indirekte Steuern wie die Mehrwertsteuer wirken schon für untere Einkommensgruppen regressiv. Haushalte mit niedrigen Einkommen müssen einen deutlich höheren Anteil ihres Einkommens für den Konsum aufbringen als Haushalte mit mittlerem oder höherem Einkommen. Diese Wirkung wird durch reduzierte Mehrwertsteuersätze etwa für Grundnahrungsmittel nur teilweise kompensiert. (Dagegen wird argumentiert, dass auch die Ersparnisse von Haushalten mit höherem Einkommen langfristig für Konsum verwendet werden - z.B. durch Erben -, womit die Mehrwertsteuer deutlich weniger regressiv wirken würde. Diese Sichtweise setzt jedoch voraus, dass akkumuliertes Vermögen auch wieder aufgezehrt wird.) Nichtsdestoweniger wirkt die Steuerfinanzierung insgesamt - verursacht vor allem durch den stark progressiven Effekt der Einkommensteuer - progressiv, während die Beitragsfinanzierung insgesamt regressiv wirkt (Härpfer et al. 2009).

Die Beschäftigungswirkungen von steuerfinanzierten im Vergleich zu beitragsfinanzierten Gesundheitsausgaben sind ebenfalls kritisch zu bewerten. Die angesprochenen Studien simulieren eine Substitution von Beitrags- durch Steuerfinanzierung. Dabei handelt es sich um Einmaleffekte, die durch eine gleichzeitige Beitragssatzsenkung und eine Steuererhöhung zur Gegenfinanzierung ausgelöst werden. Diese Einmaleffekte sind höchst sensitiv im Hinblick auf die getroffenen Annahmen in den entsprechenden Simulationen. Die empirische Überprüfung der Modellsimulationen steht noch aus.
Kritisch sind darüber hinaus die Anreizwirkungen der Steuerfinanzierung von Gesundheitsausgaben zu sehen. Es besteht die ernstzunehmende Gefahr, dass die betroffenen Akteure eine Erhöhung des Bundeszuschusses als einfachen Ausweg aus der defizitären Finanzlage der GKV ansehen. Dies gilt sowohl für Krankenkassen und Leistungserbringer als auch für die jeweils amtierende Regierung. Ein Ausbau des Steuerzuschusses ist dann bestenfalls als Ausdruck des kleinsten gemeinsamen Nenners und schlimmstenfalls als Ausdruck von Ratlosigkeit anzusehen. Gleichzeitig steigt mit dem Finanzierungsvolumen auch der staatliche Einfluss auf die Steuerung der gesundheitlichen Versorgung. Dieser geht nicht nur von Gesundheitsministerium, sondern auch vom Finanzministerium aus, z.B. in Gestalt zunehmender Aktivitäten des Bundesrechnungshofes.

Letztendlich sind die Vorteile der Steuerfinanzierung vor allem dann besonders ausgeprägt, wenn sie in Beziehung zu den Nachteilen der gegenwärtigen Beitragsfinanzierung gesetzt werden (Jacobs 2009b). Durch eine Reform der einkommensabhängigen Beitragsfinanzierung (siehe Abschnitt 3.2) würde diese der Finanzierung von Gesundheitsausgaben durch direkte Steuern jedoch deutlich ähnlicher. Mit anderen Worten: Die Vorteile einer erweiterten Beitragsbemessung und die Ausweitung des berücksichtigten Personenkreises ließen sich auch im Beitragssystem realisieren. Bis zur Realisierung des bisher vorgeschlagenen Maßnahmenbündels sollte die Steuerfinanzierung zur Vermeidung der oben angesprochenen Nachteile an verlässliche Indikatoren gebunden werden, um das erratische Auf und Ab des Bundeszuschusses zu vermeiden (Jacobs 2009a). Die Beibehaltung des Steuerzuschusses über diesen Zeitpunkt hinaus ließe sich dann nicht mehr durch die Vorteile der Steuerfinanzierung im Vergleich zur Beitragsfinanzierung, sondern allenfalls durch die Finanzierung gesamtgesellschaftlicher - in diesem Falle familienpolitischer - Aufgaben rechtfertigen (Schmähl 2006).

Der Bundeszuschuss für den Gesundheitsfonds könnte daher ähnlich wie in den Niederlanden an die Ausgaben für Kinder und Jugendliche bis zum Alter von 18 Jahren gekoppelt werden. Diese Ausgaben sind gut abgrenzbar (Jacobs 2009b), und eine Senkung des Bundeszuschusses für diese Personengruppe wäre politisch schwer durchsetzbar. Die Umverteilung zu Gunsten von Familien von Kindern erfolgt weiterhin im Beitragssystem - wird aber durch Steuermittel finanziert.

Die Forderung der privaten Krankenversicherer, dass eine solche Kopplung von Steuerzuschüssen an familienpolitische Zielsetzungen auch einen entsprechenden Zuschuss für Privatvollversicherte nach sich ziehen müsse, halten die Autoren für wenig plausibel. Kinder und Jugendliche sind in der privaten Krankenvollversicherung prämienpflichtig - es findet kein Umverteilungsmechanismus zu Gunsten von Familien statt. Insofern besteht auch kein Bedarf, einen solchen Umverteilungsmechanismus durch Steuern zu finanzieren. 


\section{THEMA}

\subsection{Reformalternative II: Strukturreform der Beitragsfinanzierung}

Die Ausweitung der Steuerfinanzierung hat einige Vorteile gegenüber dem Status quo der Beitragsfinanzierung, die vor allem in der breiteren Bemessungsgrundlage sowie in Gerechtigkeitsvorteilen liegen. Allerdings bestehen erhebliche Zweifel an der Stabilität der Steuerfinanzierung. Aus diesem Grund besteht die zweite Finanzierungsalternative darin, die Finanzierung über Beiträge insgesamt zwar „steuerähnlicher“ zu gestalten, den grundsätzlichen Charakter der Beitragsfinanzierung aber beizubehalten. Zur Verbreiterung der Bemessungsgrundlage in der Beitragsfinanzierung stehen drei Maßnahmen zur Verfügung - die Ausweitung des Versichertenkreises bzw. die Integration der privaten Krankenvollversicherung in den Risikostrukturausgleich der GKV, die Erhöhung der Beitragsbemessungsgrenze und die Verbeitragung weiterer Einkommensarten.

In der aktuellen gesundheitspolitischen Diskussion spielt die Zweiteilung des deutschen Krankenversicherungssystems in die gesetzliche und die private Krankenvollversicherung keine Rolle. Dies ist insofern bedauerlich, weil der verzerrte Systemwettbewerb zwischen GKV und PKV mindestens aus zwei Gründen zu erheblichen negativen Auswirkungen führt. Erstens schwächt die Abwanderung guter Risiken in die PKV die Stabilität und Nachhaltigkeit der GKV-Finanzierung. Zweitens führen die unterschiedlichen Vergütungssysteme insbesondere in der ambulanten ärztlichen Versorgung zu finanziellen Anreizen für die bevorzugte Behandlung von Privatversicherten (Greß 2009; Greß et al. 2009). Dabei führen die Unterschiede im Vergütungssystem im Übrigen nicht nur zur Differenzierung der Versorgung in Abhängigkeit vom Versicherungsstatus, sondern auch zu einem überproportional starken Ausgabenwachstum in der PKV - eine der zentralen Ursachen für den ungebrochenen Prämienanstieg in der privaten Krankenversicherung (Albrecht et al. 2010).

Die Versicherungspflichtgrenze hat darüber hinaus ihre Rechtfertigung längst verloren und beeinträchtigt nachhaltig auch die Effizienz im deutschen Krankenversicherungssystem. Dies ist in der nicht von der privaten Krankenversicherungswirtschaft finanzierten Wissenschaft unbestritten: „Eine Wahlmöglichkeit nach Maßgabe der Erwerbsform, sich in einer privaten oder gesetzlichen Versicherung zu versichern, lässt sich mit diesem Argument einer Schutzbedürftigkeit nicht begründen. Darüber hinaus scheint sich seit der Etablierung privater Versicherungen die Funktion der Pflichtversicherungsgrenze darauf reduziert zu haben, den privaten Versicherungen ein Marktsegment zu sichern und somit Verkrustungen bei historisch gewachsenen Strukturen im Gesundheitswesen nicht aufbrechen zu müssen“ (SVR-W 2008: Ziff. 678)“.

Gleichwohl hat die Bundesregierung in ihrem Koalitionsvertrag vereinbart, die private Krankenvollversicherung nicht anzutasten. Maßnahmen der großen Koalition, mit denen die Abwanderung von der GKV in die private Krankenvollversicherung gebremst werden sollte, sollen sogar wieder rückgängig gemacht werden (Greß 2010). Dennoch ist und bleibt die Zweiteilung des Krankenversicherungsmarktes ein zentraler Ansatzpunkt, um die Finanzierung der GKV stabiler und nachhaltiger zu gestalten, finanzielle Anreize zur bevorzugten Behandlung einzelner Personengruppen zu beseitigen und die Effizienz der Krankenversicherungssystems insgesamt zu verbessern. Deshalb ist zur Überwindung des zweigeteilten Krankenversicherungsmarktes eine einheitliche Wettbewerbsordnung einzuführen - wie es etwa in den Niederlanden 2006 mit der dortigen Krankenversicherungsreform erfolgt ist (Greß et al. 2010). Bis zur Umsetzung dieses anspruchsvollen Reformschrittes kann eine Angleichung der Vergütungsstrukturen insbesondere in der ambulanten ärztlichen Versorgung sowie die Integration der privaten Krankenvollversicherung in den Risikostrukturausgleich der GKV (vgl. Sehlen/ Schräder 2010) die negativen Auswirkungen des verzerrten Systemwettbewerbs zumindest teilweise verhindern bzw. kompensieren.

Eine zentrale Maßnahme, um die GKV-Finanzierung stabiler, nachhaltiger und gerechter zu gestalten, ist die Ausweitung der Beitragsbemessungsgrenze. Die Beitragsbemessungsgrenze ist ebenso wie die Versicherungspflichtgrenze in ihrer Festsetzung beliebig und nicht an objektive Indikatoren gebunden. Es ist nicht begründbar, warum Versicherte mit einem Einkommen oberhalb der Beitragsbemessungsgrenze relativ zu ihrem Einkommen weniger zur Finanzierung der GKV beitragen als Versicherte mit niedrigen und mittleren Einkommen (vgl. Tabelle 1). Verfassungsrechtlich unbedenklich erscheint eine Anhebung der Beitragsbemessungsgrenze - mit einer parallelen Anhebung der Versicherungspflichtgrenze - zumindest auf das Niveau der gesetzlichen Rentenversicherung (2010: 5.500 Euro im Monat in den alten Bundesländern). Mit einer deutlichen Anhebung der Beitragsbemessungsgrenze würde auch das Problem der Ungleichbehandlung zwischen Familien mit gleich hohem Haushaltseinkommen, aber unterschiedlichem Erwerbsstatus der Ehegatten zumindest ein Stück entschärft. Die verbleibenden Ungleichbehandlungen könnten durch ein Ehegattensplitting - die Anrechnung des Einkommens der erwerbstätigen Ehegatten oberhalb der Beitragsbemessungsgrenze zum Einkommen des nicht erwerbstätigen Ehegatten - gelöst werden (Dräther/Rothgang 2004). Gleichzeitig sollte auch geprüft werden, inwieweit die Beitragsbelastung niedriger Einkommen gesenkt werden könnte, und zwar sowohl aus Gründen der Verteilungsgerechtigkeit als auch zur Erhöhung der Arbeitsanreize.

Die Beitragsbemessung ist auch im Hinblick auf die berücksichtigten Einkommensarten reformbedürftig. Für pflichtversicherte Beschäftigte fallen lediglich auf Einkommen aus unselbständiger Arbeit Beiträge an. Ähnliche Regelungen gelten für Rentner. Andere Einkommensarten - insbesondere aus Kapitalvermögen - bleiben beitragsfrei. Diese enge Bemessungsgrundlage schwächt - bei einer kontinuierlich sinkenden gesamtwirtschaftlichen Lohnquote - die Stabilität und Nachhaltigkeit der Finanzierung in der GKV. Eine Verbeitragung weiterer Einkommensarten würde das Beitragsaufkommen in der GKV von der Zusammensetzung des Volkseinkommens unabhängiger machen. 
Darüber hinaus wird bisher der Grundsatz der horizontalen Gerechtigkeit - Gleichbehandlung von gleich Leistungsfähigen - verletzt (vgl. etwa das Rentner-Beispiel in Tabelle 2). Versicherte mit hohem Arbeitseinkommen und niedrigen Einkommen aus anderen Einkommensquellen werden stärker belastet als Versicherte mit niedrigem Arbeitseinkommen und hohem Einkommen aus anderen Einkommensquellen - selbst bei gleichem Gesamteinkommen.

Schon aus dem Abschlussbericht der „Rürup-Kommission “ ging hervor, dass die Einbeziehung weiterer Einkommensarten auf vielfältige praktische Erfahrungen zurückgreifen kann. Zu nennen sind hier insbesondere freiwillig versicherte Rentner und Selbständige (BMGS 2003).

In der Rürup-Kommission wurden zwei Verfahren für die Erfassung weiterer Einkommensarten vorgeschlagen. Der eine Vorschlag adaptiert das für freiwillig Versicherte von den Krankenkassen durchgeführte Verfahren, das auf der Abgabe der Einkommensteuererklärung des Vorjahres, Meldungen der Rentenversicherungsträger und der Zahlstellen von Versorgungsbezügen beruht. Interessanterweise wurde dieses Verfahren ansatzweise auch vom Bundesgesundheitsminister im Rahmen des Anfang Juni kurzzeitig propagierten Reformmodells „Gesundheitsprämie mit sozial gestaffelten Beiträgen “ aufgegriffen. Danach sollten Versicherte mit einem entsprechenden Einkommensnachweis bei den Krankenkassen einen Antrag auf einen niedrigeren Beitragssatz stellen dürfen. Explizit genannt wurden in diesem Zusammenhang Beschäftigte und Rentner mit hohen Kapitaleinkommen: „Wer beispielsweise neben seinem Lohneinkommen oder seiner Rente über hohe Zinseinkünfte verfügt, verliert den Anspruch auf Einstufung in eine niedrigere Beitragssatzklasse. Damit gewinnt das System an Gerechtigkeit (BMG 2010a: 3). "Allerdings tauchen in einer von den Versicherten bei den Krankenkassen vorzulegenden Einkommenssteuererklärung Kapitaleinkommen gar nicht auf, wenn sie der Abgeltungssteuer unterliegen. Diese „Kassenoption“ ist daher nur eingeschränkt praktikabel.

Praktikabler ist die ebenfalls von der Rürup-Kommission in die Diskussion gebrachte Poollösung. Die abzuführenden Krankenversicherungsbeträge in Höhe des vollen einkommensabhängigen Beitragssatzes werden dem Gesundheitsfonds und nicht einzelnen Krankenkassen gutgeschrieben. Das hierdurch generierte Beitragsaufkommen stabilisiert damit den einheitlichen einkommensabhängigen Beitragssatz. Die Finanzämter würden beauftragt, die insgesamt besteuerte Summe für die nicht vom Arbeitgeber erfassten Einkommensarten mit einem Beitrag für die Krankenversicherung zu belegen. Der Krankenversicherungsbeitrag wäre dann von den Finanzämtern vom Steuerpflichtigen einzuziehen und an den Gesundheitsfonds abzuführen. Analog müssten die Kreditinstitute gemeinsam mit der Abgeltungssteuer auch den entsprechenden Krankenversicherungsbeitrag einbehalten und ebenfalls an den Gesundheitsfonds abführen (BMGS 2003).

Eine verschiedentlich vorgeschlagene eigene Beitragsbemessungsgrenze etwa für Kapitaleinkommen ist jedoch abzulehnen, weil eine solche Regelung wiederum gegen den Grundsatz der horizontalen Gerechtigkeit verstieße. Versicherte, die bereits Beiträge bis zur Beitragsbemessungsgrenze entrichten, müssten sich daher bei den Kreditinstituten von der Zahlung der Krankenversicherungsbeiträge befreien lassen können.

Verschiedentlich wird kritisiert, dass die Verbeitragung weiterer Einkommensarten erhebliche Verwaltungskosten nach sich zöge. Hierauf ist jedoch zu entgegnen, dass das vorgeschlagene Verfahren bereits bestehende Einzugswege über die Finanzämter und Kreditinstitute nutzt. Außerdem steht dem durch die Verbeitragung weiterer Einkommensarten verursachten Mehraufwand - im Unterschied zu den Verwaltungskosten, die durch Zusatzbeiträge und Sozialausgleich entstehen - ein erheblicher Nutzen für die Versichertengemeinschaft gegenüber. Einerseits werden die Einmaleffekte der Verbeitragung weiterer Einkommensarten kurzfristig eine wahrnehmbare Wirkung auf die Stabilisierung des einkommensabhängigen Beitragssatzes haben. Ältere empirische Schätzungen gehen je nach Modellannahmen von einer Entlastung von bis zu 0,5 Beitragssatzpunkten aus (vgl. Tabelle 3). Der entscheidende Vorteil des Einbezugs weiterer Einkommensarten liegt andererseits aber vor allem in der mittel- bis langfristig wirksamen stärkeren Unabhängigkeit der GKV-Finanzierung von Verschiebungen in der Zusammensetzung des Volkseinkommens sowie in der größeren horizontalen Gerechtigkeit im Vergleich zum Status quo der Beitragsfinanzierung.

Tabelle 3 fasst die Ergebnisse vorliegender Studien zu den Beitragssatzeffekten einer Strukturreform der Beitragsfinanzierung zusammen. Eine sofortige Ausweitung des Versichertenkreises - bzw. der Einbezug der privaten Krankenvollversicherung in den Risikostrukturausgleich der gesetzlichen Krankenversicherung - hätte nach diesen Zahlen eine Beitragssatzentlastung von 0,7 bis 0,9 Beitragssatzpunkten zur Folge. Hier ist allerdings zu berücksichtigen, dass verfassungsrechtliche Gründe extensive Übergangsregelungen notwendig machen. Eine Ausweitung der Beitragsbemessungsgrenze für den erweiterten Versichertenkreis würde diesen Entlastungseffekt verdoppeln. Trotz teilweise leicht veralteter Zahlen wird deutlich, dass das Modell „Strukturreform der Beitragsfinanzierung“ - den entsprechenden politischen Willen vorausgesetzt - eine substantielle Option für eine „echte“ GKV-Finanzierungsreform darstellt.

\section{Literatur}

Albrecht, M./C. de Millas/S. Hildebrandt/A. Schliwen/B. Rürup (2010). Die Bedeutung von Wettbewerb im Bereich der privaten Krankenversicherungen vor dem Hintergrund der erwarteten demografischen Entwicklung. Berlin, Forschungsprojekt des Bundesministeriums für Wirtschaft und Technologie.

Albrecht, M./J. Hofmann/P. Reschke/G. Schiffhorst/S. Sehlen (2006). Stabilisierung der Finanzierungsbasis und umfassender Wettbewerb in einem integrierten Krankenversicherungssystem. Berlin, IGES.

BMG; Bundesministerium für Gesundheit (2010a). Modell einer Gesundheitsprämie mit sozial gestaffelten Beiträgen. Berlin. Juni 2010. Download: http://www.nachdenkseiten.de/upload/pdf/100604_hinweise_kopfpauschale.pdf (Zugriff: 25.07.10). 
Tabelle 3: Beitragssatzeffekte von Strukturreformen der Beitragsfinanzierung

\begin{tabular}{|l|c|c|c|}
\hline & Pfaff et al. 2006 & $\begin{array}{c}\text { Rürup-Kommission } \\
2003\end{array}$ & Albrecht et al. 2006 \\
\hline $\begin{array}{l}\text { Erhöhung der BBG in der gesetzlichen Krankenversiche- } \\
\text { rung auf das Niveau der BBG in der gesetzlichen Renten- } \\
\text { versicherung (ohne Ausweitung Versichertenkreis) }\end{array}$ & 0,4 & $0,8^{\text {a) }}$ & - \\
\hline $\begin{array}{l}\text { Verbeitragung weiterer Einkommensarten (Kapitalein- } \\
\text { kommen, Mieten, Nebeneinkünfte, private Renten) }\end{array}$ & $0,2^{\text {b) }}$ & 0,5 & - \\
\hline $\begin{array}{l}\text { Sofortige Ausweitung des Versichertenkreises (Integrati- } \\
\text { on von PKV und GKV) }\end{array}$ & 0,9 & - & 0,7 \\
\hline $\begin{array}{l}\text { Sofortige Ausweitung des Versichertenkreises (Integrati- } \\
\text { on von PKV und GKV) und Erhöhung der BBG auf das } \\
\text { Niveau in der gesetzlichen Rentenversicherung }\end{array}$ & 1,6 & 1,5 & 1,7 \\
\hline $\begin{array}{l}\text { Ausweitung des Versichertenkreises (Integration von } \\
\text { PKV und GKV) und Abschaffung der BBG }\end{array}$ & - & - & 2,7 \\
\hline
\end{tabular}

Quellen: Albrecht et al. 2006; BMGS 2003; Pfaff et al. 2006.

a) eingeschränkt vergleichbar, weil teilweise Einbeziehung von PKV (Aufhebung der Versicherungspflichtgrenze.

b) nur Kapitaleinkommen.

Alle Zahlen in Beitragssatzpunkten.

BMG, Bundesministerium für Gesundheit (2010b). Argumentationspapier zur Finanzreform für ein gerechtes, soziales, stabiles, wettbewerbliches und transparentes Gesundheitssystem. Bonn, Juli 2010. Download unter: http://www.antje-tillmann.de/politische-themen/gesundheitspolitik/index.html (Zugriff: 25.07.10).

BMGS; Bundesministerium für Gesundheit und Soziale Sicherung, Hrsg. (2003). Nachhaltigkeit in der Finanzierung der Sozialen Sicherungssysteme. Bericht der Kommission. Berlin.

Bundesregierung (2010): Für ein gerechtes, soziales, stabiles, wettbewerbliches und transparentes Gesundheitssystem („Eckpunkte-Papier“). Berlin. Juli 2010. Download unter: http://www.bmg.bund.de/cln_178/ nn_1945390/SharedDocs/Pressemitteilungen/DE/2010/pm-10-07-06gesundheitsreform.html?_nnn=true (Zugriff: 25.07.10).

Cassel, D./I. Ebsen/S. Greß/K. Jacobs/S. Schulze/J. Wasem (2008). Vertragswettbewerb in der GKV: Möglichkeiten und Grenzen vor und nach der Gesundheitsreform der Großen Koalition. Bonn, WIdO. Download unter: http://wido.de/publikation_2008.html (Zugriff: 25.07.10).

CDU/CSU/FDP (2009). Wachstum. Bildung. Zusammenhalt. Koalitionsvertrag zwischen CDU, CSU und FDP für die 17. Legislaturperiode. Berlin. Oktober 2009. Download: http://www.cdu.de/doc/pdfc/091026-koalitionsvertrag-cducsu-fdp.pdf (Zugriff: 25.07.10).

Dräther, H./H. Rothgang (2004). Die Familienversicherung für Ehepaare in der gesetzlichen Krankenversicherung. Problemanalyse und Lösungsvorschläge. Bremen, Zentrum für Sozialpolitik. ZeS-Arbeitspapier Nr. 9/2004. Download unter: http://www.zes.uni-bremen.de/ccm/content/ veroeffentlichungen/arbeitspapiere.de?id=218 (Zugriff: 25.07.10).

Ebsen, I. (2010). Verfassungsrechtliche Anforderungen des Gleichheitsgrundsatzes (Art. 3 Abs. 1 GG) an die Ausgestaltung des Sozialausgleichs im Rahmen der geplanten gesetzlichen Änderungen beim kassenindividuellen einkommensunabhängigen Zusatzbeitrag zur GKV. Eine Einschätzung des verfassungsrechtlichen Risikos ungleicher Berücksichtigung beitragspflichtiger Einnahmen auf Bitte des Parteivorstands der SPD. Mainz. Juli 2010.

Greß, S. (2009). Mit gleichen Rahmenbedingungen zu einem fairen Wettbewerb im Gesundheitssystem. Zur Notwendigkeit einer einheitlichen Wettbewerbsordung auf dem deutschen Krankenversicherungsmarkt. WISO direkt - Analysen und Kozepte zur Wirtschafts- und Sozialpolitik. Bonn, Friedrich-Ebert-Stiftung.

Greß, S. (2010). Strategiewechsel in der Gesundheitspolitik schafft mehr Probleme als Lösungen. Aushöhlung des Solidaritätsprinzips - Einseitige Belastung der Versicherten - Noch mehr Klientelpolitik. WISO diskurs - Expertisen und Dokumentationen zur Wirtschafts- und Sozialpolitik. Bonn, Expertise im Auftrag des Gesprächskreises Sozialpolitik der Friedrich-Ebert-Stiftung.

Greß, S./S. Leiber/M. Manouguian (2009). Integration von privater und gesetzlicher Krankenversicherung vor dem Hintergrund internationaler Erfahrungen. WSI-Mitteilungen 62 (7): 369-375.

Greß, S./S. Heinemann/K. Jecobs (2010). Gesundheitsreform: Spicken beim Nachbarn. Gesundheit und Gesellschaft 13 (1): 20-26.

Härpfer, M./M. Cacace/H. Rothgang (2009). And Fairness for all? Wie gerecht ist die Finanzierung im deutschen Gesundheitssystem? Eine Berechnung des Kakwani-Index auf Basis der EVS. Bremen, Zentrum für Sozialpolitik. ZeS-Arbeitspapier Nr. 4/2009. Download unter: http:// www.zes.uni-bremen.de/ccm/content/veroeffentlichungen/arbeitspapiere.de?id=281 (Zugriff: 25.07.10)

Häussler, B./T. Ecker/M. Schneider (2006). Belastung der Arbeitgeber in Deutschland durch gesundheitssystembedingte Kosten im internationalen Vergleich. Baden-Baden, Nomos.

Jacobs, K. (2008). Der Morbiditätsbezug des RSA als Voraussetzung für versorgungsorientierten Kassenwettbewerb. In: D. Göpffarth/S. Greß/ K. Jacobs/J. Wasem (Hrsg.): Jahrbuch Risikostrukturausgleich 2008: Morbi-RSA. Sankt Augustin, Asgard: 133-152.

Jacobs, K. (2009a). Ordnungspolitische Defizite der Gesundheitspolitik. Gesundheits- und Sozialpolitik 63 (3): 11-16.

Jacobs, K. (2009b). Ring frei zur nächsten Runde. Eine „echte“ Finanzierungsreform ist weiter überfällig. G+G Wissenschaft 9 (3): 13-22. Download: http://wido.de/fileadmin/wido/downloads/pdf_ggw/ wido_ggw_aufs2_0709.pdf (Zugriff: 25.07.10).

Jacobs, K./P. Reschke/D. Cassel/J. Wasem (2002). Zur Wirkung des Risikostrukturausgleichs in der gesetzlichen Krankenversicherung. Schriftenreihe des Bundesministeriums für Gesundheit Bd. 140. Baden-Baden, Nomos.

Meinhardt, V./R. Zwiener (2005). Gesamtwirtschaftliche Wirkungen eine Steuerfinanzierung versicherungsfremder Leistungen in der Sozialversicherung. Berlin, DIW. Download: http://diw.de/documents/ publikationen/73/diw_01.c.43075.de/diwkompakt_2005-007.pdf (Zugriff: 25.07.10).

Monopolkommission (2010). Mehr Wettbewerb, wenig Ausnahmen. 18 Hauptgutachten der Monopolkommission gemäß § 44 Abs. 1 Satz 1 GWB. Download: http://www.monopolkommission.de/haupt_18/ mopoko_volltext_h18.pdf (Zugriff: 25.07.10). 
Pfaff, A. B./M. Pfaff/B. Langer/F. Manberer/F. Freund/N. Holl (2006). Finanzierungsalternativen in der Gesetzlichen Krankenversicherung. Einflussfaktoren und Optionen zur Weiterentwicklung. Berlin, edition sigma.

Reiners, H. (2009). Mythen der Gesundheitspolitik. Bern, Huber.

Schawo, D. (2007). Gesundheitsfonds und Einkommensausgleich zwischen den Krankenkassen. In: D. Göpffarth/S. Greß/K. Jacobs/J. Wasem (Hrsg.): Jahrbuch Risikostrukturausgleich 2007: Gesundheitsfonds. Sankt Augustin, Asgard: 97-114.

Schmähl, W. (2006). Aufgabenadäquate Finanzierung der Sozialversicherung durch Beiträge und Steuern - Begründungen und Wirkungen eines Abbaus der „Fehlfinanzierung“ in Deutschland. Bremen, Zentrum für Sozialpolitik. ZeS-Arbeitspapier Nr. 5/2006. Download unter: http:// www.zes.uni-bremen.de/ccm/content/veroeffentlichungen/arbeitspapiere.de?id=251 (Zugriff: 25.07.10).

Sehlen, S/W. F. Schräder (2010). Risikostrukturausgleich innerhalb der PKV und zwischen PKV und GKV nach dem Wettbewerbsstärkungsgesetz. In: D. Göpffarth/S. Greß/K. Jacobs/J. Wasem (Hrsg.): Jahrbuch Risikostrukturausgleich 2009/10: Von der Selektion zur Manipulation? Heidelberg, medhochzwei: 93-114.
SVR-W; Sachverständigenrat zur Begutachtung der gesamtwirtschaftlichen Entwicklung (2008). Die Finanzkrise meistern, Wachstumskräfte stärken. Jahresgutachten 2008/09. Bundestags-Drucksache 16/10985 vom 18.11.2008. Download: http://dip21.bundestag.de/dip21/ btd/16/109/1610985.pdf (Zugriff: 25.07.10).

Van de Ven, W. P. M. M./F. T. Schut/H. E. G. M. Hermans/J. D. de Jong/M van der Maat/R. Coppen/P. P. Groenewegen/R. D. Friele (2009). Evaluatie Zorgverzekeringswet en Wet op de zorgtoeslag. Den Haag, ZonMW. Programma evaluatie regelgeving: deel 27.

Wasem, J./S. Greß/K. Jacobs (2007). Gesundheitsfonds und Regionaldebatte I: Ordnungspolitischer Rahmen. In: D. Göpffarth/S. Greß/K. Jacobs/J. Wasem (Hrsg.): Jahrbuch Risikostrukturausgleich 2007: Gesundheitsfonds. Sankt Augustin, Asgard: 139-162.

Wille, E. (2010). Die Finanzierungsbasis der gesetzlichen Krankenversicherung - Reformen unumgänglich. Gesundheitsökonomie und Qualitätsmanagement 15 (3): 102-104.

\section{Gesundheitsstandort privater Haushalt}

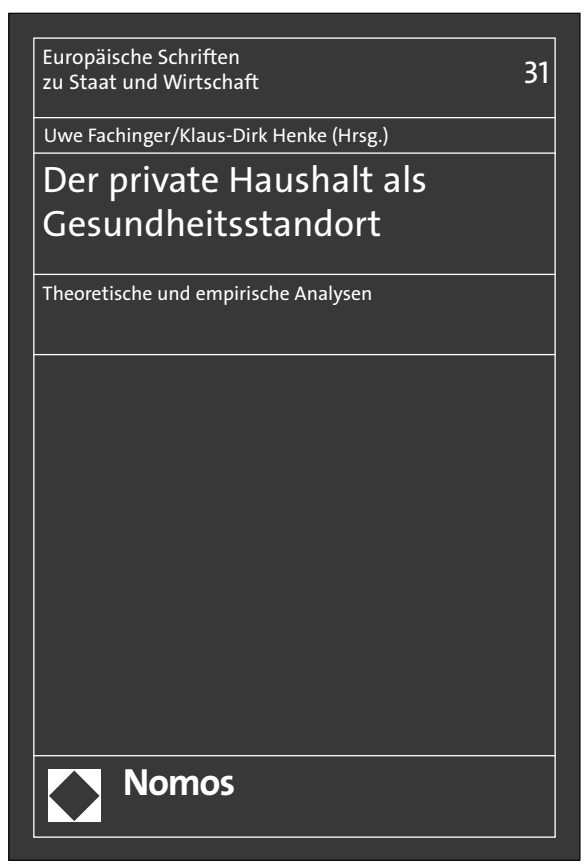

\section{Der private Haushalt als \\ Gesundheitsstandort}

Theoretische und empirische Analysen

Herausgegeben von Univ. Prof. Dr. Uwe Fachinger und

Prof. Dr. Klaus-Dirk Henke

2010, 240 S., brosch., 59,-€, ISBN 978-3-8329-5718-6

(Europäische Schriften zu Staat und Wirtschaft, Bd. 31)

Der private Haushalt wird sich durch den zunehmenden Einsatz von assistierenden Technologien und Ambient Assisted LivingSystemen (AAL) als Gesundheitsstandort herausbilden. In den Beiträgen werden die damit verbundenen ökonomischen Aspekte insbesondere im Zusammenhang mit der gesundheitlichen und pflegerischen Versorgung älterer Menschen behandelt.

Bitte bestellen Sie im Buchhandel oder

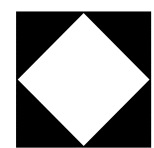

